\title{
Prevention of Medication-related Osteonecrosis of the Jaw (MR-ONJ) after dentoalveolar surgery in cancer patients: one year experience.
}

\author{
Giorgia Capocasale ${ }^{1}$, Fabio Lonardi ${ }^{1}$, Annarita Signoriello ${ }^{1}$, giorgio panozzo, Francesca Zotti ${ }^{1}$, Riccardo Nocini ${ }^{1}$, \\ Massimo Albanese ${ }^{1}$ \\ 1 University of Verona
}

Funding: The author(s) received no specific funding for this work.

Potential competing interests: The author(s) declared that no potential competing interests exist.

\section{Abstract}

Medication-related Osteonecrosis of the Jaw (MR-ONJ) is an adverse drugs reaction characterized by the worsening of quality of life in cancer patients, and for which one of the most frequent risk factor recognized is tooth extraction.

This report aims to describe our one-year experience about the prevention of MR-ONJ in cancer patients receiving antiresorptive-antiangiogenic drugs, with or without Bisphosphonates, referred to the Department of Surgical Sciences, Paediatrics and Gynaecology (University of Verona).

The protocol described by the Italian Society of Oral and Maxillofacial Surgery (SICMF) and the Italian Society of Oral Pathology and Medicine (SIPMO) was strictly followed in all surgical procedures, including the defined medical prophylaxis.

Once assessed inclusion and exclusion criteria, a retrospective analysis was carried out in a total of 15 patients, followed up during 2019. None of our patients developed MR-ONJ.

\section{INTRODUCTION}

Bone metastasis, frequently found in patients with advanced solid tumors stages, is reported as the third most common site of metastasis ${ }^{[1]}$. Moreover, cancer therapies may result in a higher risk of bone loss, subsequently enhancing the occurring of skeletal-related events (SREs) in these patients ${ }^{[2]}$.

To limit these bone-related major adverse effects, the employment of pharmacologic therapies known as Antiresorptive drugs (Bisphosphonates and Denosumab) and Antiangiogenic drugs (e.g. Vascular Endothelial Growth

Factor inhibitors such as Bevacizumab, Tyrosine Kinase inhibitors such as Sunitinib and mammalian Target of Rapamycin inhibitors such as Everolimus) ${ }^{[3]}$ was proposed.

A potential adverse drugs reaction associated with the administration of the mentioned above drugs is Medication-related Osteonecrosis of the Jaw (MR-ONJ) ${ }^{[4]}$ : this adverse reaction worsens the quality of life in cancer patients. At this proposal, one of the most frequent risk factor widely recognized is tooth extraction.

As aetiology of MR-ONJ is multifactorial and its correct patho-physiology remains unknown ${ }^{[5]}$, definitive protocols on its 
prevention in oral surgery has not been established yet.

This report aims to describe our one-year experience with the prevention of MR-ONJ in cancer patients referred to our Department for tooth extractions, and receiving antiresorptive-antiangiogenic drugs with or without Bisphosphonates.

\section{MATERIALS AND METHODS}

A retrospective analysis was carried out on patients referred to the Section of Dentistry and Maxillofacial Surgery of University of Verona (Italy), during 2019; patients were selected for the present study if they had exposure to Antiresorptive and/or Antiangiogenic drugs. We excluded patients with history of radiation therapy for the craniofacial region and patients receiving only Bisphosphonates.

After the assessment of inclusion/exclusion criteria, the following information were researched in medical records and collected:

1. Patient demographics (age, gender)

2. Kind of cancer

3. Type of Antiresorptive and/or Antiangiogenic drugs treatment

4. Other drugs and systemic diseases

5. Oral local risk factors.

The protocol described and proposed by the Italian Society of Oral and Maxillofacial Surgery (SICMF) and the Italian Society of Oral Pathology and Medicine (SIPMO) ${ }^{[6]}$ was followed to perform the tooth extractions.

This protocol includes medical prophylaxis and a strictly described surgical procedure:

- Antibiotic treatment consisting in amoxicillin + clavulanic acid $(875 \mathrm{mg}+125 \mathrm{mg})$ three times per day and metronidazole (500mg) three times per day. This antibiotic therapy was administered from the day before the surgical procedure and for at least 6 days following intervention.

- $0,12 \%$ Chlorhexidine mouthwash 3 times per day for 15 days; growth-promoting treatment, as gel containing hyaluronic acid, three times per day for 15 days.

- Surgical procedure included local anesthesia without adrenaline, atraumatic extraction of the tooth, alveoloplasty (if necessary), and tension-free soft tissue closure, to allow healing by first intention.

For assessing the effectiveness of the protocol, we decided to considered only surgical procedures were performed by training surgeons under the supervision of a senior surgeon.

Timing of checks was scheduled as follows for all patients:

- $t_{0}$ (baseline): first visit, which included general health assessment, accurate general anamnesis about previous pathologies, allergies, previous surgeries, radiotherapy, current and past drug treatments.

- $t_{1}$ (the day of dental extraction): the timing of surgical procedures was established considering the SIPMO guidelines.

Described surgical procedures were performed for each patient.

- $t_{2}$ (2 weeks after dental extraction), $t_{3}$ ( 1 month follow-up), $t_{4}$ (2 months follow-up): follow-up visits at each time-point provided complete intra and extra oral examination performed by the same surgeon.

According to the SIPMO guidelines, the restarting of MR-ONJ therapy was recommended 4 or 6 weeks after dental extraction. 


\section{RESULTS}

A total of 15 patients ( 4 females and 11 males) met the inclusion and exclusion criteria. Mean age was 64,4 years, for a range between 56 and 77 years.

Metastatic renal cell carcinoma was the most common diagnosis (40\%), followed by breast (20\%), Multiple Myeloma $(13,33 \%)$, prostate cancer, melanoma, colon cancer and gastric cancer $(6,67 \%)$.

Concerning the MR-ONJ therapy, the following drugs were taken: Denosumab by 7 patients, Sunitinib by 4 patients, Bevacizumab by 2 patients and Everolimus by 2 patients. This treatment was respectively associated with Zoledronate, chemotherapy drugs (such as Thalidomide, Nivolumab, Paclitaxel) and corticosteroids in 5, 10 and 3 patients.

Moreover, general medical histories showed positivity for systemic diseases including hypertension, dyslipidemia, thyroid gland disorder, depression.

The clinical and surgical protocol was applied to all patients, and all surgical procedures were performed by training surgeons.

The most frequent local risk factors (found in $80 \%$ of cases) were complications related to periapical abscess for failed endodontic treatment or to endoperiodontal abscess. Three patients presented teeth with baleful prognosis because of destructive caries lesions.

None of our patients presented MR-ONJ at each follow-up recall medical examinations.

\section{CONCLUSION}

MR-ONJ is a problematic oral complication in cancer patients, that extensively worsens their quality of life ${ }^{[7]}$. In addition, in some cases, we should consider the possibility of ONJ onset in cancer patients in palliative therapy, for which surgical treatment of this complication could be difficult to perform ${ }^{[8]}$.

In this contest, oral professionals assume a key role in the prevention of this adverse drug reaction.

This retrospective study aims to describe our one-year experience with the prevention of MR-ONJ in cancer patients who needed tooth extractions. As the usual method, we followed the protocol described and proposed by the SICMF and the SIPMO. For this investigation we considered only surgical procedures performed by training surgeons. According to these conditions, our results showed that none of our patients developed MR-ONJ. Then, these results could proved that the followed protocol could be applied with excellent results also by training surgeons.

Another outcome can be underlined considering the reasons which led to dental extractions: in $80 \%$ of cases, a relation with periapical abscess for failed endodontic treatment or endo-periodontal abscess was demonstrated. Specifying that all patients examined were subjected to dental examination at their dentists, it could be hypothesized that an important issue could take into account an previous dental treatments, more specifically inadeguate endodontic therapies.

Even though our outcomes did not demonstrate any cases of MR-ONJ, these are preliminary data: as the study sample is very limited, prospective approaches with larger number of patients are desirable, with possible caution in the evaluation of re-treatment, instead of extraction, of teeth presenting inadequate endodontic therapies at the first visit. 


\section{References}

1. `Jaime Fornetti, Alana L Welm, Sheila A Stewart. (2018). Understanding the Bone in Cancer Metastasis. J Bone Miner Res, vol. 33 (12), 2099-2113. doi:10.1002/jbmr.3618.

2. `Soojung Hong, Taemi Youk, Su Jin Lee, Kyoung Min Kim, et al. (2020). Bone metastasis and skeletal-related events in patients with solid cancer: A Korean nationwide health insurance database study. PLoS ONE, vol. 15 (7), e0234927. doi:10.1371/journal.pone.0234927.

3. `Katlynn M. Mathis, Kathleen M. Sturgeon, Renate M. Winkels, Joachim Wiskemann, et al. (2018). Bone resorption and bone metastasis risk. Medical Hypotheses, vol. 118, 36-41. doi:10.1016/j.mehy.2018.06.013.

4. 'Nouf A AlDhalaan, Asma BaQais, Ahmad Al-Omar. (2020). Medication-related Osteonecrosis of the Jaw: A Review. doi:10.7759/cureus.6944.

5. ^S. Ruggiero, D. Saxena, S. Tetradis, T. Aghaloo, et al. (2018). Task Force on Design and Analysis in Oral Health Research: Medication-Related Osteonecrosis of the Jaw. JDR Clinical \& Translational Research, vol. 3 (3), $222-225$. doi:10.1177/2380084418770662.

6. ^Olga Di Fede, Vera Panzarella, Rodolfo Mauceri, Vittorio Fusco, et al. (2018). The Dental Management of Patients at Risk of Medication-Related Osteonecrosis of the Jaw: New Paradigm of Primary Prevention. BioMed Research International, vol. 2018 , 1-10. doi:10.1155/2018/2684924.

7. 'Gianluca Tenore, Ahmed Mohsen, Antonella Francesca Rossi, Gaspare Palaia, et al. (2020). Does MedicationRelated Osteonecrosis of the Jaw Influence the Quality of Life of Cancer Patients?. Biomedicines, vol. 8 (4), 95. doi:10.3390/biomedicines8040095.

8. 'Massimo Albanese, Francesca Zotti, Giorgia Capocasale, Stefano Bonetti, et al. (2020). Conservative non-surgical management in medication related osteonecrosis of the jaw: A retrospective study. Clin Exp Dent Res, vol. 6 (5), $512-$ 518. doi:10.1002/cre2.303. 